\title{
Skin metabolism established with the use of MetaSite for selected retinoids employed in topical and systemic treatment of various skin disorders and found in cosmeceuticals
}

\author{
Karolina Słoczyńska1凶 ${ }^{\circledR}$, Agnieszka Gunia-Krzyżak², Dorota Żelaszczyk², \\ Anna M. Waszkielewicz ${ }^{2}$ and Henryk Marona ${ }^{2}$
}

1Department of Pharmaceutical Biochemistry, 2Department of Bioorganic Chemistry, Chair of Organic Chemistry, Faculty of Pharmacy, JagielIonian University Medical College, Kraków, Poland

\begin{abstract}
Purpose. Besides being widely used in cosmetics, retinoids are potent therapeutic agents used topically and systemically as anti-acne agents. The aim of this study was to predict with the use of MetaSite the skin metabolism of selected retinoids employed in treatment of skin disorders and found in cosmeceuticals. The following compounds were studied: retinol, retinaldehyde, retinoic acid, retinyl acetate, retinyl palmitate, acitretin, etretinate, adapalene and bexarotene. Methods. MetaSite, Molecular Discovery Ltd. is a computational model that enables prediction of cytochrome P450-dependant metabolism. This software indicates atoms in the molecule structure that are mostly vulnerable to metabolic changes and predicts the metabolite structures. Results. MetaSite indicated that retinol and retinal metabolites were obtained through hydroxylation of the methyl group located in the position 3 of the aliphatic chain, whereas retinoic acid biotransformation would occur principally in the carbon atom situated in the position 4 in the cyclohexene ring. In acitretin molecule, carbon atom of the methoxy group attached to the benzene ring displayed the highest probability of biotransformation. In etretinate, metabolic reactions would occur principally on the carbon atom of the final ethyl group of the molecule. Conclusions. MetaSite metabolism predictions for retinoic acid, acitretin, etretinate, adapalene and bexarotene were in agreement with experimental findings. In case of compounds being converted by catalysts other than cytochrome P450 enzymes, the primary metabolites predicted by MetaSite differ from those reported previously. In conclusion, MetaSite is a useful tool that can aid identification of the major metabolites of compounds being administered topically.
\end{abstract}

Key words: cytochrome P450, MetaSite, retinoids, skin metabolism Received: 10 September, 2014; revised: 11 February, 2015; accepted: 13 February, 2015; available on-line: 09 April, 2015

\section{INTRODUCTION}

The term retinoids refers to vitamin A (retinol, ROL) and its natural and synthetic derivatives. Through interactions with specific cellular and nucleic acid receptors this group of compounds influences many vital biological processes such as regulation of skin function and neuronal development (Sorg et al., 1999; Hellmann-Re- gen et al., 2013). Besides being widely used in cosmetic products, retinoids are potent therapeutic agents used topically and systemically as highly effective anti-acne agents. A hallmark of endogenous retinoid signaling in the skin is its local, paracrine, homeostatic regulation, in which local retinoic acid (RA) metabolism plays an essential role (Baron et al., 2008).

It was demonstrated previously that retinoids antagonize reduced cell growth and increased collagen-degrading matrix metalloproteinases in naturally aged human skin and regulate the keratinization process (Varani et al., 1990; Baron et al., 2005; Jean et al., 2011). Additionally, retinoids abolish sun-induced skin hyperpigmentation and are effective in treatment of the sun-damaged skin (Nyirady et al., 2001; Draelos, 2005; Kang et al., 2005; Mukherjee et al., 2006; Sorg et al., 2006; Serri \& Iorizzo, 2008; Kircik, 2012; Hubbard et al., 2014). Moreover, these compounds stimulate the production of collagen and elastin in the dermis. This is achieved through transformation of less active fibroblasts into cells that produce large amounts of collagen (Varani et al., 2000; Rossetti et al., 2011). The increase in the number and activity of fibroblasts improves skin firmness, elasticity and hydration. Additionally, retinoids may be also classified as anti-inflammatory agents, inhibiting e.g. microglial activation (Hellmann-Regen et al., 2013).

Retinoids are divided into four categories based on their chemical structure. First generation includes the natural, nonaromatic compounds. Retinoids belonging to this group such as ROL, RA and isotretinoin are used in the treatment of acne. The second generation of retinoids consists of monoaromatic compounds that are synthetic vitamin A analogs. This group of compounds is useful in the pharmacotherapy of severe forms of psoriasis and keratinization disorders (Ianhez et al., 2013). In this category there are acitretin and etretinate (Sarkar et al., 2013). The third generation such as adapalene, bexarotene and tazarotene are polyaromatic retinoid derivatives and are used in the therapy of plaque psoriasis. Finally, fourth generation of retinoids comprises pyranones such as seletinoid $G$ (Blomhoff \& Blomhoff, 2006; Mukherjee et al., 2006; Brun et al., 2013). Seletinoid $G$ is a novel synthetic retinoid that was found to repair

e-mail: karolina.sloczynska@uj.edu.pl

Abbreviations: ARAT, acyl CoA: acyltransferase; CYP450, cytochrome P450; LRAT, lecithin: retinol-acyltransferase; M1-3, metabolites 1-3; PS, probability score; $R A$, retinoic acid; $R A L$, retinaldehyde; $R A L D H$, retinal dehydrogenase; $R A L R$, retinal reductase; $R D H$, retinol dehydrogenase; $\mathrm{REH}$, retinyl esters hydrolase; $\mathrm{ROL}$, retinol; SOM, site of metabolism 
altered connective tissue and to inhibit UV-induced collagen deficiency when tested in aged human skin in vivo (Kim et al., 2005).

With respect to their skin action, retinoids can be divided into three categories i.e. topically administered retinoids registered as drugs (e.g. tretinoin, isotretinoin, alitretinoin, tazarotene and adapalene); systemic retinoids that are not available for topical treatment (e.g. acitretin and etretinate); and compounds incorporated into skin products (e.g. ROL, retinaldehyde (RAL) and retinyl esters (REs)) (Babamiri \& Nassab, 2010; Sarkar et al., 2013; Hubbard et al., 2014).

As the skin is the active site of retinoid metabolism, the aim of this study was to predict the local (skin) metabolism of selected retinoids, employed for topical and systemic treatment of various skin disorders and found in cosmeceuticals, by using the MetaSite computational technique. The following compounds were studied: ROL, RAL, RA, retinyl acetate, retinyl palmitate, acitretin, etretinate, adapalene and bexarotene. Subsequently, the results obtained were compared to experimental observations in order to evaluate the utility of MetaSite in drug discovery metabolite identification studies.

\section{MATERIALS AND METHODS}

MetaSite, Molecular Discovery Ltd., v.3.1.1.2 is a computational model that enables prediction of the cytochrome P450 (CYP450)-dependent metabolism in phase I biotransformations. The main idea of MetaSite methodology is analysis of the protein-ligand interactions. This software is primarily designed to indicate atoms in the molecular structure that are the most vulnerable to metabolic changes due to the cytochrome action. These regions are known as the sites of metabolism (SOM) or hot spots. Validation procedures have proved that in $85 \%$ of the analyzed structures the indicated metabolic sites agreed in top three rankings with experimental data (Cruciani et al., 2005; Molecular Discovery Ltd., 2014). Additionally, the method predicts atoms in the molecular structure that contribute to a particular SOM. Another important MetaSite advantage is prediction of chemical structures of the most likely metabolites (Zhou et al., 2006).

The probability of a specific atom $i$ to be a site of metabolism catalyzed by the heme moiety of CYP450 depends on several factors, namely the accessibility of atom $i$ towards the heme (indicated as Ei); the chemical reactivity of atom $i$ in the specific reaction mechanism (described as $\mathrm{R} \imath$ ) and the relative probability of a reaction mechanism under consideration that occurs (called $\mathrm{M} i)$. Thus, the probability function $\left(\mathrm{P}_{\mathrm{SM}}\right)$ for a specific atom $i$ can be defined by the following equation: $\mathrm{P}_{\mathrm{SM} i}=$ E $i \times \mathrm{R} i \times \mathrm{M} i$ (Cruciani et al., 2005; Molecular Discovery Ltd., 2014). Lastly, the final ranking for potential metabolic sites is obtained and the probability score (PS) is given.

It is noteworthy that in silico tools such as MetaSite that predict the metabolism of xenobiotics may be useful in the assessment of a compound's metabolic stability and therefore, may enable the synthesis of new compounds with improved metabolic profile. The metabolism prediction is usually performed for the most prevalent human CYP450 isoforms such as 1A2, 2C9, 2C19, 2D6 and 3A4. Thus, a comprehensive prediction for specific organs such as liver, skin and brain is provided.

\section{RESULTS}

The MetaSite metabolic predictions for all analyzed compounds are presented in Fig. 1. The top three sites of metabolism were presented for each tested compound.

In case of both ROL (Fig. 1A) and RAL (Fig. 1B) MetaSite predicted that the carbon atom of the methyl group located in position 3 of the aliphatic chain was most likely metabolized by the skin (retinol PS of 12.5 ; RAL PS of 10.5). The software calculations predicted that the primary ROL and RAL metabolites (M1) were obtained through the hydroxylation at the methyl group located in this particular position. M2 and M3 of RAL and M3 of ROL were the products of aliphatic carbonylation that lead to the formation of carbonyl (ketone) derivatives of ROL and RAL. Finally, M2 of ROL is a derivative formed via dehydrogenation with vinyl substituent in ROL aliphatic chain.

The prediction of RA (Fig. 1C) metabolism showed that its biotransformation would occur principally at the carbon atom located in position 4 in cyclohexene ring (PS of 6.7). The secondary SOM was a methyl substituent in positions 7 (PS of 6.1) and 3 (PS of 5.8) of the aliphatic chain. That skin enzymes would catalyze aliphatic hydroxylation of RA was the most probable biotransformation pathway that produced three main RA metabolites such as 4-hydroxyretinoic acid (4-OH-RA) (M1), cyclohexa-1,3-diene derivative (M2) and 4-oxoretinoic acid (4-oxo-RA) (M3).

In retinyl acetate molecule (Fig. 1D) MetaSite indicated the carbon atom situated in position 4 in cyclohexene ring as the prime metabolic position (PS of 8.8), whereas the atoms located in positions 3 and 7 of the aliphatic chain as the second and the third SOM (PS of 8.1 and 7.9 , respectively). The primary three retinyl acetate metabolites were generated through aliphatic hydroxylation leading to the formation of 4-hydroxy-(M1), 4-oxo(M2), and cyclohexa-1,3-diene derivatives (M3) of retinyl acetate.

In case of retinyl palmitate (Fig. 1E) MetaSite gave the highest metabolism priority to the carbon atom in position 15 located in the ester fragment derived from palmitic acid (PS of 8.8), whereas the second and the third SOM were methyl groups in positions 3 and 7 of the aliphatic ester fragment derived from retinol (PS of 8.1 and 7.9, respectively). The main metabolic reaction indicated by the software was aliphatic hydroxylation that lead primarily to the formation of retinyl 15-hydroxypalmitate (M1). Additionally, M2 and M3 were the products of aliphatic hydroxylation and vinylic dehydrogenation at the methyl substituent located in position 3 of retinyl aliphatic fragment of the molecule.

Prediction of the metabolic fate of acitretin (Fig. 1F) showed that the carbon atom of methoxy group attached to the benzene ring displayed the highest PS of biotransformation (10.1); next in the hierarchy was the carbon atom located at the methyl substituent in position 7 of the aliphatic chain (PS of 6.4), and finally the carbon atom located in position 3 on benzene ring (PS of 5.4). The main metabolite of acitretin was generated through $\mathrm{O}$-dealkylation within methoxy group substituted in a ring, whereas the other two metabolites possessed hydroxyl group or carbonyl at the methyl group in position 7 of the aliphatic chain of acitretin.

The primary site of CYP attack predicted by MetaSite for etretinate (Fig. 1G) was at the carbon atom of the final ethyl group of the molecule, where biotransformation started with the PS of 13.3. Next in hierarchy was 
<smiles>CC(C=CC1=C(C)C2(C)CCCCC2C1(C)C)=CC=CC(C)=CCO</smiles><smiles>CC1=C(/C=C/C(C)=C/C=C/C(=C\CO)CO)C(C)(C)CCC1</smiles>

M1 $(\log P=4.71)$<smiles>C=C(/C=C/O)/C=C/C=C/C(C)=C/C=C/C1=C(C)CCCC1C</smiles>

M2 $(\log P=7.32)$

B

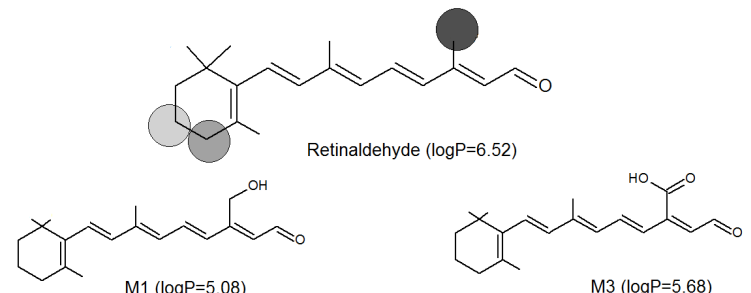<smiles>CC1=C(C)C(/C=C/C(C)=C/C=C/C(C=O)=C/C=O)=C(C)CCC1</smiles>

C<smiles>[Y]C1CC=CC(C)=C1C=CC=C(C)C</smiles>

D

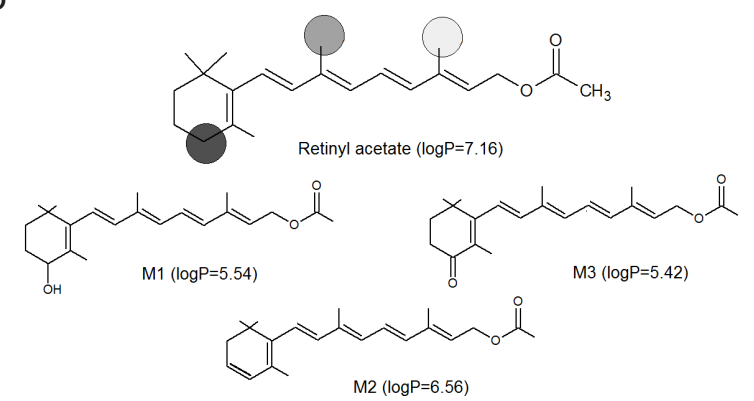

E

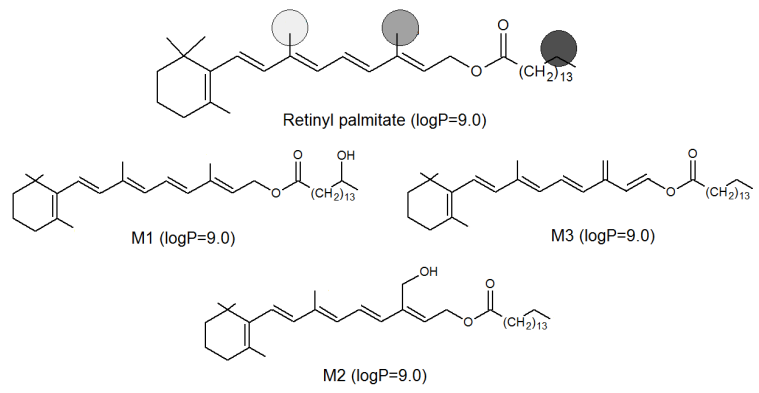<smiles>CC(/C=C/C=C1/C=CC=C1/C=C/c1c(C)cc(Oc2ccccc2)c(C)c1-c1ccccc1)=C\C(=O)O</smiles><smiles>COc1cc(C)c(/C=C/C(C=O)=C/C=C/C(C)=C/C(=O)O)c(C)c1C</smiles><smiles>COc1cc(C)c(/C=C/C(=C/C=C/C(C)=C/C(=O)O)CO)c(C)c1C</smiles>

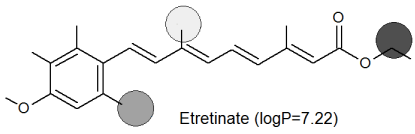<smiles>C=COC(=O)/C=C(C)/C=C/C=C(C)/C=C/c1c(C)cc(OC)c(C)c1C</smiles>

$M 1(\log P=6.28)$ M3 $(\log P=6.97)$<smiles>CC(=O)OCCOCCO</smiles>

$\mathrm{H}$

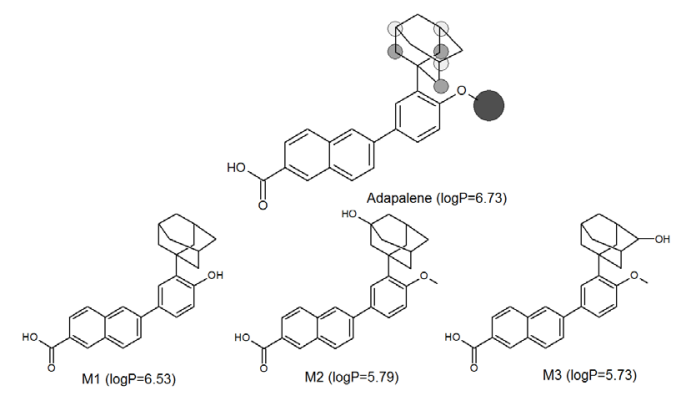

I

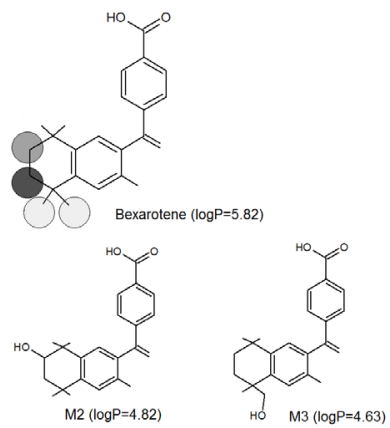

Figure 1A-I. Plots of MetaSite predictions for sites of metabolism of the analyzed compounds.

The functional groups in compounds that most likely will be metabolized by skin tissue are marked: the darker the color the higher the probability score (PS) of metabolism to occur; M1-3metabolites. LogP values calculated using MetaSite are given in brackets. 
the methyl substituent carbon atom attached to the benzene ring in position 6 (PS of 7.6) and carbon atom at the methyl group in position 7 of the aliphatic chain (PS of 6.3). Two primary metabolites of etretinate i.e. acitretin and acetic acid were generated through O-dealkylation, whereas M3 was the product of dehydrogenation.

The software assigned the carbon atom of the methoxy group located in position 4 of the phenyl ring in adapalene (Fig. $1 \mathrm{H})$ as the most vulnerable to skin metabolic changes (PS of 8.2). Further sites of metabolism were indicated within adamantyl moiety (PS of 5.8). In case of adapalene the first metabolic reaction was $O$ dealkylation, whereas the next two were aliphatic hydroxylations. Due to metabolic biotransformation adapalene hydroxy metabolites or dealkylated products were formed.

The last compound tested was bexarotene, where calculations indicated that its biotransformation (Fig. 1I) started at the carbon atom located in position 3 of the dihydronaphthalene ring (PS of 9.1). The carbon atom situated in position 7 of the same ring was indicated as the second SOM (PS of 4.8). Finally, carbon atoms of methyl groups attached to dihydronaphthalen in position 5 were predicted as the third SOM (PS of 4.7). Aliphatic hydroxylation was the metabolic reaction that gave three main bexarotene metabolites, namely 6-hydroxy, 7-hydroxy and 5-hydroxymethyl-5-methyl- derivatives of bexarotene.

\section{DISCUSSION}

In silico models are useful tools to study metabolic fate of chemical compounds. In the present study we used MetaSite, a metabolism site prediction program, to predict CYP450-derived skin metabolites of selected retinoids used in the treatment of various skin conditions as well as found in skin care products. The compounds investigated were ROL, RAL, RA, retinyl acetate, retinyl palmitate, acitretin, etretinate, adapalene and bexarotene. The results obtained were compared to experimental observations in order to evaluate the utility of MetaSite in drug discovery metabolite identification studies.

It was demonstrated previously that under physiological conditions RA is synthesized via the RDHs (retinol dehydrogenases)/RALDHs (retinal dehydrogenases) and metabolically inactivated via the CYP450 enzymes, especially 26A, 26B, but also by 1A2, 3A4, 2C8, 2C9 and 2C19 (Roos et al., 1998; Blomhoff \& Blomhoff, 2006; Oesch et al., 2007; Baron et al., 2008; von Lintig, 2012; Brun et al., 2013; Conaway et al., 2013). That is why predicting the metabolic fate of retinoids is of such great relevance.

MetaSite prediction of aliphatic hydroxylation and aliphatic carbonylation of RA as the most probable pathways of biotransformation proved to be accurate as it was reported previously that (4-OH-RA) and (4-oxo$\mathrm{RA}$ ) are the most important RA metabolites. Both compounds are removed from the cells more rapidly than the parent compound (Westin et al., 1993; Duell et al., 1996; Sorg et al., 1999). It is noteworthy that MetaSite portrayed 3,4-didehydroretinoic acid as one of the most probable products of RA metabolism (Reynolds et al., 1993).

Derivatives of ROL such as retinyl acetate and retinyl palmitate are characterized by increased chemical stability as compared to ROL. Therefore, REs are widely used in cosmetics. Upon presentation of REs to MetaSite, the software indicated aliphatic hydroxylation of both molecules as the most likely route of metabolism resulting in appropriate hydroxy derivatives. Additional retinyl acetate metabolites were cyclohexa-1,3-diene- and 4-oxoretinyl acetate, whereas retinyl palmitate in silico biotransformation gave metabolites with hydroxy group substituted in the cyclohexene ring or in the aliphatic chain. To the best of our knowledge, no such metabolites have been observed in vivo/ in vitro as REs primarily metabolic transformation is hydrolysis to ROL (Roos et al., 1998; Sorg et al., 2006). This reaction is catalyzed by retinyl ester hydrolases (REHs) (Törmä \& Vahlquist, 1990; O’Byrne \& Blaner, 2013). It was demonstrated that in human skin, $44 \%$ of the absorbed retinyl palmitate is hydrolyzed to ROL (Boehnlein et al., 1994).

Topical ROL is a precursor of cutaneous RAL as RDHs and retinal reductase (RALR) enable the reversible conversion of ROL and RAL. Next, RAL is irreversibly oxidized into $\mathrm{RA}$ in reactions catalyzed by RALDH and CYP450 enzymes (Kurlandsky et al., 1994; Roos et al., 1998; O’Byrne \& Blaner, 2013). Then, after the conversion to appropriate REs, ROL is stored in the skin (Sorg et al., 2006). This reaction is possible due to the presence of lecithin: retinol-acyltransferase (LRAT) and acyl CoA: acyl-transferase (ARAT) (Kurlandsky et al., 1996; O’Byrne \& Blaner, 2013). Subsequently, REH catalyzes the hydrolysis of REs to ROL, thus providing the new pool of active ROL. On the other hand, topical RAL is mostly biotransformed into ROL and REs, and only a small amount of retinal undergoes oxidation to RA. CYP450 enzymes are involved in RAL conversion to 4-hydroxyretinal (Siegenthaler et al., 1990; Sorg et al., 1999; Mukherjee et al., 2006). According to MetaSite, both ROL and RAL molecules undergo primarily aliphatic hydroxylation to generate appropriate hydroxy metabolites. Additionally, the software indicated carbonyl (ketone) derivatives of ROL and RAL.

With reference to the second generation of retinoids, our in silico tool indicated that aromatic retinoid acitretin metabolic fate was related to O-dealkylation that lead to the formation of appropriate hydroxy metabolites. In silico metabolism indicated also the formation of carbonyl metabolites of acitretin. At the same time, MetaSite suggested that acitretin is the primary metabolite of etretinate. Such a metabolic pathway is a valuable result as etretinate is indeed converted to acitretin and may serve as a prodrug for acitretin (Millikan, 2005; Sarkar et al., 2013).

In case of the third generation of retinoids, adapalene in silico biotransformation gave appropriate hydroxy metabolites or dealkylated products of the parent compound. Adapalene is a derivative of naphthoic acid, which has a non-steroidal anti-inflammatory agent (NSAID)-like structure, but retinoid-like features. It is also worthwhile noting that in case of topically applied adapalene its metabolic fate is actually unknown and its metabolites were not identified. It was stated, however, that its biotransformation involves primarily O-demethylation and hydroxylation of the methoxy benzene ring of adapalene and this is consistent with our in silico prediction (Ayala, 2006). Finally, 6-hydroxybexarotene was predicted as the most probable metabolite of bexarotene. This was confirmed experimentally when bexarotene metabolism was studied using animal liver slices and pooled human microsomes (Howell et al., 2001).

In case of all compounds tested, except for retinyl palmitate, the primary metabolite's $\log \mathrm{P}$ values were lower than those of the parent compound. This is with accordance with general rule that catabolism of chemical compounds generates polar water-soluble metabolites. 
It is rather due to the pharmacodynamic effects (severe side effects) that retinoid's topical application is preferred over systemic application. Pharmacokinetic effects are always an issue with retinoids, which massively induce their own metabolism via upregulation of CYP450 enzymes. Therefore, a full view of the metabolism of topically applied compounds is of great importance. Obviously, in vitro and in vivo experiments are irreplaceable, however, there is a need for the use of in silico predictions to get answers on a given compound's metabolism. The main advantages of in silico models vs. in vitro and in vivo experiments are their simplicity, relatively low cost and the possibility to make fast predictions for a large group of chemical compounds even before the synthesis of a particular compound. Thus, compounds presenting an unfavorable (e.g. toxic) metabolic profile would not be synthesized. On the other hand, the main drawbacks of in silico methods with respect to in vitro and in vivo metabolism models are related to the fact that the expert systems are based on the combination of metabolic rules appropriate for various mammalian models (species), consequently in silico models may not reflect species specific pathways and reliable metabolism prediction (Kirchmair et al., 2012). Additionally, the software tools tend to generate a large amount of metabolites which may hinder the classification and prioritization of predictions (Langowski \& Long, 2002; Kirchmair et al., 2012).

After analyzing retinoid metabolism data collected by using MetaSite it can be concluded that for some compounds such as RA, acitretin, etretinate, adapalene and bexarotene the predictions were very accurate and metabolites indicated by the software are in agreement with experimental findings. For other compounds, namely ROL, RAL, retinyl acetate and retinyl palmitate a discrepancy existed between in silico prediction and data demonstrated in the literature that is based on in vitro and in vivo models. Such difference is attributed to the fact that MetaSite applies only to specific enzymes such as those belonging to CYP450 family, that are the most important enzymes responsible for xenobiotics metabolism in human skin (Oesch et al., 2007; Svensson, 2009). Therefore, in case of retinoids being converted by biological catalysts other than CYP450 enzymes or by CYP enzymes that are not part of the MetaSite database, such as e.g. CYP26A1 which is responsible for the metabolism of RA in the skin cells, the primary metabolites predicted by software differ from those reported in the literature. In order to obtain a more comprehensive view of a given compound's metabolism, several specific models present in various software should be combined. In conclusion, MetaSite predictive expert system is a very useful tool that can aid the identification of major metabolites of compounds being administered topically.

\section{REFERENCES}

Ayala FA (08.08.2006) Patent US20090318371 A1, Topical antiacne preparations containing retinoid (tazarotene or adapalene), antibiotic (clindamycin phosphate) and/or keratolytic (microsponged benzoyl peroxide).

Babamiri K, Nassab R (2010) Cosmeceuticals: the evidence behind the retinoids. Aesthet Surg J 30: 74-77.

Baron JM, Heise R, Blaner WS, Neis M, Joussen S, Dreuw A, Marquardt Y, Saurat JH, Merk HF, Bickers DR, Jugert FK (2005) Retinoic acid and its 4-oxo metabolites are functionally active in human skin cells in vitro. J Invest Dermatol 125: 143-153.

Baron JM, Wiederholt T, Heise R, Merk HF, Bickers DR (2008) Expression and function of cytochrome p450-dependent enzymes in human skin cells. Curr Med Chem 15: 2258-2264.

Boehnlein J, Sakr A, Lichtin JL, Bronaugh RL (1994) Characterization of esterase and alcohol dehydrogenase activity in skin. Metabolism of retinyl palmitate to retinol (vitamin A) during percutaneous absorption. Pharm Res 11: 1155-1159.

Blomhoff R, Blomhoff HK (2006) Overview of retinoid metabolism and function. I Neurobiol 66: 606-630.

Cruciani G, Carosati E, De Boeck B, Ethirajulu K, Mackie C, Howe T, Vianello R (2005) MetaSite: understanding metabolism in human cytochromes from the perspective of the chemist. J Med Chem 48: 6970-6979.

Brun PJ, Yang KJ, Lee SA, Yuen JJ, Blaner WS (2013) Retinoids: Potent regulators of metabolism. Biofactors 39: 151-163.

Conaway HH, Henning P, Lerner UH (2013) Vitamin a metabolism, action, and role in skeletal homeostasis. Endocr Rev 34: 766-797.

Draelos ZD (2005) Novel approach to the treatment of hyperpigmented photodamaged skin: $4 \%$ hydroquinone $/ 0.3 \%$ retinol versus tretinoin 0.05\% emollient cream. Dermatol Surg 31: 799-804.

Duell EA, Kang S, Voorhees JJ (1996) Retinoic acid isomers applied to human skin in vivo each induce a 4-hydroxylase that inactivates only trans retinoic acid. I Invest Dermatol 106: 316-320.

Hellmann-Regen J, Kronenberg G, Uhlemann R, Freyer D, Endres M, Gertz K (2013) Accelerated degradation of retinoic acid by activated microglia. I Neuroimmunol 15: 1-6.

Howell SR, Shirley MA, Grese TA, Neel DA, Wells KE, Ulm EH (2001) Bexarotene metabolism in rat, dog, and human, synthesis of oxidative metabolites, and in vitro activity at retinoid receptors. Drug Metab Dispos 29: 990-998.

Hubbard BA, Unger JG, Rohrich RJ (2014) Reversal of skin aging with topical retinoids. Plast Reconstr Surg 133: 481e-490e.

Ianhez M, Fleury LF Jr, Miot HA, Bagatin E (2013) Retinoids for prevention and treatment of actinic keratosis. An Bras Dermatol 88: $585-593$.

Jean J, Soucy J, Pouliot R (2011) Effects of retinoic acid on keratinocyte proliferation and differentiation in a psoriatic skin model. Tissue Eng. Part A 17: 1859-1868.

Kang S, Duell EA, Fisher GJ, Datta SC, Wang ZQ, Reddy AP, Tavakkol A, Yi JY, Griffiths CE, Elder JT, Voorhees JJ (1995) Application of retinol to human skin in vivo induces epidermal hyperplasia and cellular retinoid binding proteins characteristic of retinoic acid but without measurable retinoic acid levels or irritation. I Invest Dermatol 105: 549-556.

Kang S, Bergfeld W, Gottlieb AB, Hickman J, Humeniuk J, Kempers S, Lebwohl M, Lowe N, McMichael A, Milbauer J, Phillips T, Powers J, Rodriguez D, Savin R, Shavin J, Sherer D, Silvis N, Weinstein R, Weiss J, Hammerberg C, Fisher GJ, Nighland M, Grossman R, Nyirady J (2005) Long-term efficacy and safety of tretinoin emollient cream $0.05 \%$ in the treatment of photodamaged facial skin: a two-year, randomized, placebo-controlled trial. Am J Clin Dermatol 6: $245-253$.

Kim MS, Lee S, Rho HS, Kim DH, Chang IS, Chung JH (2005) The effects of a novel synthetic retinoid, seletinoid $G$, on the expression of extracellular matrix proteins in aged human skin in vivo. Clin Chim Acta 362: 161-169.

Kirchmair J, Williamson MJ, Tyzack JD, Tan L, Bond PJ, Bender A, Glen RC (2012) Computational prediction of metabolism: sites, products, SAR, P450 enzyme dynamics, and mechanisms. J Chem Inf Model 52: 617-648.

Kircik LH (2012) Safety and efficacy evaluation of tretinoin cream $0.02 \%$ for the reduction of photodamage: a pilot study. J Drugs Dermatol 11: 83-90.

Kurlandsky SB, Xiao JH, Duell EA, Voorhees JJ, Fisher GJ (1994) Biological activity of all-trans retinol requires metabolic conversion to all-trans retinoic acid and is mediated through activation of nuclear retinoid receptors in human keratinocytes. J Biol Chem 269: 32821-32827.

Kurlandsky SB, Duell EA, Kang S, Voorhees JJ, Fisher GJ (1996) Auto-regulation of retinoic acid biosynthesis through regulation of retinol esterification in human keratinocytes. J Biol Chem 271: $15346-15352$.

Langowski J, Long A (2002) Computer systems for the prediction of xenobiotic metabolism. Adv Drug Deliv Rev 54: 407-415.

Millikan LE (2005) Drug Therapy in Dermatology (Basic and Clinical Dermatology). Taylor \& Francis e-library.

Molecular Discovery Ltd., http://www.moldiscovery.com (06.05.2014).

Mukherjee S, Date A, Patravale V, Korting HC, Roeder A, Weindl G (2006) Retinoids in the treatment of skin aging: an overview of clinical efficacy and safety. Clin Interv Aging 1: 327-348.

Nyirady J, Bergfeld W, Ellis C, Levine N, Savin R, Shavin J, Voorhees JJ, Weiss J, Grossman R (2001) Tretinoin cream $0.02 \%$ for the treatment of photodamaged facial skin: a review of 2 double-blind clinical studies. Cutis 68: 135-142.

O’Byrne SM, Blaner WS (2013) Retinol and retinyl esters: biochemistry and physiology. I Lipid Res 54: 1731-1743.

Oesch F, Fabian E, Oesch-Bartlomowicz B, Werner C., Landsiedel R (2007) Drug-metabolizing enzymes in the skin of man, rat, and pig. Drug Metab Rev 39: 659-698.

Reynolds NJ, Fisher GJ, Griffiths CE, Tayakkol A, Talwar HS, Rowse PE, Hamilton TA, Voorhees JJ (1993) Retinoic acid metabolites 
exhibit biological activity in human keratinocytes, mouse melano$\mathrm{ma}$ cells and hairless mouse skin in vivo. J Pharmacol Exp Ther 266: 1636-1642.

Roos TC, Jugert FK, Merk HF, Bickers DR (1998) Retinoid metabolism in the skin. Pharmacol Rev 50: 315-333.

Rossetti D, Kielmanowicz MG, Vigodman S, Hu YP, Chen N, Nkengne A, Oddos T, Fischer D, Seiberg M, Lin CB (2011) A novel anti-ageing mechanism for retinol: induction of dermal elastin synthesis and elastin fibre formation. Int J Cosmet Sci 33: 62-69.

Sarkar R, Chugh S, Garg VK (2013) Acitretin in dermatology. Indian J Dermatol Venereol Leprol 79: 759-771.

Serri R, Iorizzo M (2008) Cosmeceuticals: focus on topical retinoids in photoaging. Clin Dermatol 26: 633-635.

Siegenthaler G, Saurat JH, Ponec M (1990) Retinol and retinal metabolism. Relationship to the state of differentiation of cultured human keratinocytes. Biochem J 268: 371-378.

Sorg O, Didierjean L, Saurat JH (1999) Metabolism of topical retinaldehyde. Dermatology 199 (Suppl 1): 13-17.

Sorg O, Antille C, Kaya G, Saurat JH (2006) Retinoids in cosmeceuticals. Dermatol Ther 19: 289-296.

Svensson CK (2009) Biotransformation of drugs in human skin. Drug Metab Dispos 37: 247-253.
Törmä H, Vahlquist A (1990) Vitamin A esterification in human epidermis: a relation to keratinocyte differentiation. J Invest Dermatol 94 : 132-138.

Varani J, Mitra RS, Gibbs D, Phan SH, Dixit VM, Mitra R Jr, Wang T, Siebert KJ, Nickoloff BJ, Voorhees JJ (1990) All-trans retinoic acid stimulates growth and extracellular matrix production in growth-inhibited cultured human skin fibroblasts. J Invest Dermatol 94: 717-723.

Varani J, Warner RL, Gharaee-Kermani M, Phan SH, Kang S, Chung JH, Wang ZQ, Datta SC, Fisher GJ, Voorhees JJ (2000) Vitamin A antagonizes decreased cell growth and elevated collagen-degrading matrix metalloproteinases and stimulates collagen accumulation in naturally aged human skin. J Invest Dermatol 114: 480-486.

von Lintig J (2012) Metabolism of carotenoids and retinoids related to vision. J Biol Chem 287:1627-1634.

Westin S, Mode A, Murray M, Chen R, Gustafsson JA (1993) Growth hormone and vitamin A induce P4502C7 mRNA expression in primary rat hepatocytes. Mol Pharmacol 44: 997-1002.

Zhou D, Afzelius L, Grimm SW, Andersson TB, Zauhar RJ, Zamora I (2006) Comparison of methods for the prediction of the metabolic sites for CYP3A4-mediated metabolic reactions. Drug Metab Dispos 34: 976-983. 